\title{
A posição horizontal entre cliente e psicólogo na Abordagem Centrada na Pessoa
}

\author{
The horizontal position between client and psychologist in the Person-Centered Approach \\ La posición horizontal entre cliente y psicólogo en el Enfoque Centrado en la Persona
}

Recebido: 03/01/2022 | Revisado: 09/01/2022 | Aceito: 15/01/2022 | Publicado: 17/01/2022

\author{
Sabrina Andrade Rocha \\ ORCID: https://orcid.org/0000-0003-0939-7975 \\ Clínica de Psicologia Sabrina Andrade, Brasil. \\ E-mail: sabrina.andrade.rocha@gmail.com \\ Marciana Gonçalves Farinha \\ ORCID: https://orcid.org/0000-0002-2024-7727 \\ Universidade Federal de Uberlândia, Brasil \\ E-mail: farinhamarciana@gmail.com
}

\begin{abstract}
Resumo
O objetivo deste estudo foi compreender a posição horizontal e não-hierárquica entre psicoterapeuta e cliente dentro da Abordagem Centrada na Pessoa na clínica psicológica. Para isto, foi feita uma pesquisa bibliográfica qualitativa que visou responder esse objetivo. Foram utilizados os seguintes descritores: 'clínica psicológica', 'contexto histórico', 'Abordagem Centrada na Pessoa'. Os artigos utilizados foram encontrados em bases de dados como: Scientific Electronic Library Online (SciELO) e Portal de Periódicos Eletrônicos de Psicologia (PePSIC). A clínica, na Abordagem Centrada na Pessoa, tem suas particularidades em detrimento das outras abordagens terapêuticas, entre elas a que mais se destaca é a sua visão de ser humano. Para a Abordagem Centrada na Pessoa, o ser humano possui a capacidade inata de buscar seu desenvolvimento desde que possua as condições necessárias para isto. Este é o conceito de tendência atualizante de Carl Rogers. Desta forma, não precisaria que na relação terapêutica alguém guiasse o cliente, pois o psicoterapeuta crê em sua autonomia como indivíduo. A tendência atualizante seria então o alicerce desta próspera ligação entre psicoterapeuta e cliente, que não busca ensinar algo, mas facilitar para que o cliente desenvolva suas potencialidades.
\end{abstract}

Palavras-chave: Clínica; Humanismo; Psicoterapia.

\begin{abstract}
The aim of this study was to understand the horizontal and non-hierarchical position between psychotherapist and client within the person-centered approach in psychological clinic. For this, a qualitative bibliographic research was carried out that aimed to answer this objective. The following descriptors were used: psychological clinic, historical context, Person-Centered Approach. The articles used were found in databases such as: Scientific Electronic Library Online (SciELO) and Portal of Electronic Psychology Journals (PePSIC). The clinic in the Person-Centered Approach has its particularities to the detriment of other therapeutic approaches, among which the one that stands out most is its vision of being human. For the Person Centered Approach, the human being has the innate capacity to seek his development as long as he has the necessary conditions for this, this is the concept of Carl Rogers' current trend. Thus, he would not need someone to guide him in the therapeutic relationship, as the psychotherapist believes in his autonomy as an individual. The current trend would then be the foundation of this prosperous connection between psychotherapist and client, who does not seek to teach something but to facilitate the client to develop their potential.

Keywords: Clinic; Humanism; Psychotherapy.

\section{Resumen}

El objetivo de este estudio fue comprender la posición horizontal y no jerárquica entre el psicoterapeuta y el cliente dentro del enfoque centrado en la persona en la clínica psicológica. Para ello, se realizó una investigación bibliográfica cualitativa que tuvo como objetivo dar respuesta a este objetivo. Se utilizaron los siguientes descriptores: clínica psicológica, contexto histórico, enfoque centrado en la persona. Los artículos utilizados fueron encontrados en bases de datos como: Scientific Electronic Library Online (SciELO) y el Portal de Revistas de Psicología Electrónica (PePSIC). La clínica en el Enfoque Centrado en la Persona tiene sus particularidades en detrimento de otros enfoques terapéuticos, entre los que destaca la visión del ser humano. Para el Enfoque Centrado en la Persona, el ser humano tiene la capacidad innata de buscar su desarrollo siempre que tenga las condiciones necesarias para ello, este es el concepto de tendencia de actualización de Carl Rogers. De esta forma, no necesitaría que alguien lo guiara en la relación terapéutica, pues el psicoterapeuta cree en su autonomía como individuo. La tendencia actualizada sería entonces la base de esta conexión próspera entre el psicoterapeuta y el cliente, que no busca enseñar algo, sino facilitar que el cliente desarrolle su potencial.
\end{abstract}

Palabras clave: Clínica; Humanismo; Psicoterapia. 


\section{Introdução}

As relações de poder que permeiam a palavra clínica são tão antigas quanto o próprio conceito de cuidado. De acordo com Moreira et al. (2007), o começo da atividade clínica (do grego klinê/leito) envolve o médico, que na cabeceira do enfermo, faz um exame dos sintomas da doença para elaborar um diagnóstico, um prognóstico e prescrever um tratamento.

Um marco da Psicologia Clínica ocorreu no ano de 1896 e o primeiro a mencionar este conceito foi Lightner Witmer que criou a primeira clínica com a nomenclatura de Psicologia Clínica, na Universidade da Pensilvânia, desenvolvendo um caminho profissional para o psicólogo neste campo. Desta forma, o papel de Lightner Witmer foi fundamental, pois por meio de sua criação foi possível demarcar de maneira conceitual qual seria a área em desenvolvimento da psicologia clínica. Nesta data também ocorre outro evento significativo, pois Freud nomeia de psicanálise o seu processo clínico, mudando o foco do biológico para um conhecimento firmado na escuta biográfica, no qual é possível uma compreensão adequadamente psicológica, junto com psicopatologia e psicoterapia (Moreno, 2014).

A Abordagem Centrada na Pessoa - ACP nasce como uma contraposição às teorias psicanalítica e comportamentalista, ou seja, este saber propõe uma visão diferente de homem. Segundo Rogers (1992), "o indivíduo existe num mundo de experiências em constante mutação, do qual ele é o centro" (p. 549). Este mundo é denominado de campo fenomenológico, que se refere ao mundo singular de cada um, constituído por todas as experimentações vividas pelo indivíduo, sendo elas percebidas conscientemente ou não.

A relação clínica psicológica na Abordagem Centrada na Pessoa tem suas particularidades tendo em vista as outras abordagens terapêuticas. Uma delas é compreender o cliente como alguém que possui uma tendência que o impulsiona ao seu próprio desenvolvimento quando possui as condições adequadas para isto. Sendo assim, a tendência atualizante é uma das bases desta relação não-hierárquica entre cliente e psicólogo.

O objetivo deste estudo foi compreender a posição horizontal e não-hierárquica entre psicoterapeuta e cliente na abordagem centrada na pessoa na clínica psicológica. Para tanto, foi realizada uma revisão bibliográfica de cunho qualitativo.

\section{Metodologia}

Considerando o objetivo deste trabalho, desenvolvemos um estudo de cunho qualitativo e bibliográfico. Foi realizada uma revisão bibliográfica narrativa. Nas pesquisas qualitativas, principalmente nos casos em que não se possui um modelo teórico para análise, é comum observar um movimento entre observações, reflexões e interpretações na proporção que a análise tem andamento. A pesquisa bibliográfica é elaborada a partir de um conteúdo já formulado que se constitui essencialmente de livros e artigos científicos (Gil, 2002).

Foram utilizados os seguintes descritores: 'clínica psicológica', 'contexto histórico' e 'Abordagem Centrada na Pessoa'. Os artigos utilizados foram encontrados em bases de dados como: Scientific Electronic Library Online (SciELO) e Portal de Periódicos Eletrônicos de Psicologia (PePSIC). Não foi realizado recorte temporal. Foram consultados apenas livros e artigos que correspondessem ao objetivo deste estudo.

\section{Contexto Histórico da Palavra 'Clínica' e suas Relações de Poder}

Inicialmente, é importante entender o significado do conceito da palavra clínica, para em seguida compreender seu trajeto histórico. Moreira et al. (2007) destacam que o começo da atividade clínica envolve o médico que, na cabeceira do enfermo, faz um exame dos sintomas da doença para elaborar um prognóstico, um diagnóstico e recomendar um possível tratamento. Para isto, o médico se utiliza de entrevistas e observações. Estes processos, originalmente nos sugerem uma reflexão a respeito da prioridade do saber médico sobre o saber psicológico. 
No conhecimento em que se baseia a práxis médica, não é possível diagnosticar antes que seja feita a descrição dos sintomas e assim o histórico da doença. Da mesma forma, é impossível realizar um prognóstico antes de possuir um diagnóstico. No entanto, não foi sempre assim. Anteriormente a Hipócrates, a prática médica estava mais aproximada do âmbito mágico do que da racionalidade. Há 2.500 anos, Hipócrates promoveu mudanças na Medicina da Grécia com a intenção de entender o contexto da doença no paciente e o que levaria este paciente a buscar um tratamento (Moreira et al., 2007).

Com Hipócrates, inaugurou-se a análise clínica e foi criada a anamnese, determinando estes procedimentos como a primeira fase do atendimento médico. O exame médico foi por ele inserido na clínica, com o objetivo de obter informações para o diagnóstico e o prognóstico. O exame médico criado por Hipócrates era formado por um conjunto de procedimentos, entre eles: observar cuidadosamente, medir a temperatura por meio da imposição das mãos, apalpar o corpo e auscultar os batimentos cardíacos (Moreira et al., 2007).

O ápice da clínica médica se situa entre o final do século XVIII e o início do século XIX. O século XIX foi, com certeza, um dos mais produtivos para a medicina, em razão das várias descobertas na área da Biologia e as criações que permitiram instrumentalizar os aparatos médicos. Foucault (1977) cria uma anarquia histórica ao expor o início da clínica no fim do século XVIII e início do XIX. Na realidade, a clínica médica surge com Hipócrates; o que acontece neste período é o progresso da clínica devido aos meios técnicos nos diagnósticos (Moreira et al., 2007).

Seguindo a análise histórica da clínica, no século XX, a medicina junta os saberes adquiridos e consolidados com as novas tecnologias, o que transforma a prática médica. A inclusão de dispositivos diagnósticos, as várias opções de tratamentos avançados e de nível elevado de especificidade médica levam a seguinte ideia: quanto mais complexa a questão, mais fundamental é se especializar. A partir deste período, a clínica médica se divide em vários fragmentos. Então, passa a atribuir ao paciente a função de decidir qual especialista procurar, uma vez que houve uma compartimentação do saber e cada profissional vê uma parte do indivíduo. A prática do cuidar vira ofício mais detalhado, o que altera os costumes; agora quem vai ao médico é o enfermo (Moreira et al.,2007).

Sendo assim, Moreira et. al. (2007) levantam estas reflexões sobre a clínica: teria acontecido um contra-movimento da posição de médico e paciente? Será que devido a esta especialização dos saberes não houve um distanciamento da visão humanista que também é elementar na Medicina? Mesmo que estas reflexões sejam fundamentais e intrigantes da visão da clínica de uma forma geral, são importantes apenas para uma contextualização antes de adentrar as questões da clínica psicológica.

\section{A clínica psicológica e suas relações com o indivíduo}

A clínica psicológica em seu contexto histórico é cercada por marcos que ao decorrer dos anos moldaram seu curso e sua forma contemporânea. Freud nomeia de psicanálise o seu processo clínico, mudando o foco que antes assenta no biológico para um conhecimento firmado na escuta biográfica, no qual é possível uma compreensão psicológica, junto com psicopatologia e psicoterapia (Moreno, 2014). Outro evento marcante é a consolidação da psicologia clínica como uma profissão e a criação da Associação Americana de Psicologia - APA, em 1892 (Trull \& Phares, 2003).

A data de início da Psicologia Clínica foi o ano de 1896. O primeiro indivíduo que mencionou este conceito foi Lightner Witmer que criou a primeira Clínica de Psicologia, na Universidade da Pensilvânia, criando um caminho profissional para o psicólogo neste campo (Gonzáles, 2008). Desta forma, o papel de Witmer, foi essencial, pois por meio de sua criação foi possível demarcar de maneira conceitual a área em desenvolvimento da psicologia clínica (Compas \& Gotlib, 2003).

A clínica psicológica é a atividade profissional mais almejada entre os alunos de psicologia nos primórdios do curso e, na atualidade, ainda congrega muitos adeptos (Magalhães et al., 2001). Na busca por um caminho com respostas para a 
indagação: 'o que é clínica?', após realizar uma contextualização do nascimento etimológico e histórico da palavra 'clínica', é importante pensar sobre as combinações entre a Psicologia e clínica (Moreira et al., 2007).

Uma visão usualmente aceita é de que a construção da atitude clínica de um discente de psicologia está firmada em três aspectos principais: entendimento teórico, supervisão da prática clínica e psicoterapia pessoal. Estes aspectos são intrinsecamente relacionados, sendo que o conhecimento teórico só é integrado e compreendido durante a psicoterapia pessoal que faz possível o autoconhecimento e o uso de habilidades pessoais na compreensão e análise dos processos psíquicos. E, a supervisão, ainda, procura auxiliar na incorporação destes dois fatores na prática clínica, sendo eles o autoconhecimento e o conhecimento teórico. Inferimos, por ato clínico, a perspectiva de se estabelecer na função profissional incorporado de uma demarcação específica, conservando empatia com o cliente. A conduta clínica possibilita firmar uma ligação de respeito com o outro e delimita as atitudes éticas que o profissional deve seguir, ao mesmo tempo que este procura uma elaboração sobre o que ocorre com o cliente (Aguirre et al., 2000).

\section{A Abordagem Centrada na Pessoa de Carl Rogers}

Nesta abordagem psicológica, a atuação do psicoterapeuta é ser um facilitador para seu cliente durante a psicoterapia e seu progresso. O termo 'cliente' que Rogers (1992) decidiu adotar foi para substituir o termo 'paciente' que emite o entendimento de passividade. Assim sendo, a terapia é centrada na busca do cliente e, em seu ritmo, não diretiva e não sendo conduzida pelo terapeuta.

A Abordagem Centrada na Pessoa foi inovadora no seu tempo e serviu até mesmo de inspiração para Rosenberg desenvolver a Comunicação não-violenta (Calabria \& Kempfer,2020).

A vivência se determina com o que ocorre no organismo a todo momento. Isto acontece no campo fenomenal e perceptual incluindo fatos inconscientes e conscientes simbolizados. Desta forma, para se entender a ideia de experiência nos conceitos da ACP é preciso falar sobre o campo fenomenológico.

Rogers (1992) destaca que "o indivíduo existe num mundo de experiências em constante mutação, do qual ele é o centro" (p. 549). Este mundo é denominado de campo fenomenológico, que se refere ao mundo singular de cada um, constituído por todas as experimentações vividas pelo indivíduo, sendo elas percebidas conscientemente ou não. Ele ainda menciona que estas vivências são conscientes quando são elaboradas e/ou simbolizadas, ou seja, quando se escreve e se pensa sobre estas vivências é que o sujeito que faz delas cientes à consciência, por isso, apenas algumas experiências são conscientemente vividas.

É essencial salientar que no campo fenomenológico, exclusivamente, o próprio sujeito sabe o sentido autêntico e completo do seu mundo de vivências. Somente ele conhece como a experiência foi compreendida. Por este motivo, Rogers (1992) declara que o conhecimento e a consciência do campo fenomenológico possuem limitações. Uma limitação do campo fenomenológico seria que ele se constitui como uma forma subjetiva do indivíduo se ver e de enxergar o mundo, construindo sua própria percepção do que é real.

Toda conduta é relativa à noção de realidade percebida e significada pelo campo fenomenológico. Um exemplo disso seria quando existe uma realidade compartilhada entre vários indivíduos percebendo um mesmo objeto, no entanto, cada pessoa não percebe aquela realidade em si e sim a sua própria percepção (Rogers, 1992).

Rogers (1983) explicita que existe uma tendência atualizadora em todos indivíduos. Esta tendência é inerente ao organismo e o impulsiona a crescer, desenvolver e atualizar suas potencialidades e recursos numa direção autônoma construtiva. Estas potencialidades podem ser despertadas se houver um clima de atitudes facilitadoras. 
Para alcançar os resultados esperados, não é necessário técnica, mas atitudes, pois o enfoque está na capacidade do cliente e não somente no conhecimento do terapeuta. O psicólogo americano se referia a três atitudes ou condições facilitadoras para o crescimento pessoal do cliente: autenticidade/congruência, aceitação positiva incondicional e empatia.

Por autenticidade, entende-se uma postura verdadeira na sua percepção e sentimentos na relação com o cliente que busca a ajuda (Araujo \& Freire, 2014). A congruência consiste em ser transparente, de maneira que suas palavras estejam em concordância com seus sentimentos e representações subjetivas dentro da relação, no caso, a terapêutica. Já a aceitação positiva incondicional é uma aceitação do outro como uma pessoa de valor, entendendo que este individuo está procurando se encontrar, independentemente de sua condição, de suas atitudes, e ou sentimentos. É a aceitação das atitudes expressas no momento, sem ser preciso considerar o quão negativas ou positivas elas são. A empatia se refere à compreensão empática quando o terapeuta deve perceber com solicitude as significações pessoais e os sentimentos que o cliente vivencia, comunicando a ele sua compreensão e a disponibilidade de ajuda-lo. Estar disposto a suspender seus princípios e valores, para que tenha condições de não julgar e direcionar a ajuda terapêutica (Moreira \& Torres, 2013).

A Psicoterapia foi introduzida no campo de trabalho do psicólogo devido os esforços de Rogers, nos Estados Unidos, que antes praticava aconselhamento. A atividade do aconselhamento esteve inicialmente vinculada a várias possibilidades de ação, entre elas: encorajamento, feedback positivo, orientação, interpretação e fornecimento de informações (ScorsoliniComin, 2014). Rogers posteriormente foi fazer uma psicoterapia semelhante ao aconselhamento, porém, mais intenso, sendo esta prática profissional exportada para o Brasil (Amatuzzi, 2012).

A ACP orienta que a psicoterapia é um dos tipos de auxílio psicológico e pode ser utilizada para além da psicologia e em variados campos de atuação humana ou em qualquer atividade que tenha relação com ser humano. Portanto, a psicoterapia centrada na pessoa é, assim, a aplicação da ACP em psicoterapia a fim de promover seu desenvolvimento pessoal. O foco é o indivíduo em seus sentimentos e intenções, sendo ele mesmo o responsável pelo seu processo de desenvolvimento pessoal por meio do auxílio do psicólogo na facilitação deste processo. Deste modo, lança mão de suas atitudes de acolhimento, compreensão empática, estando aberto ao novo e ao diferente, no contato direto com o cliente com foco em dizer uma palavra que o faça pensar, evidenciando sua preocupação para com ele e o levando a um processo de encontro consigo mesmo (Amatuzzi, 2012).

Para Rogers, o mais importante na psicoterapia são as atitudes e a disposição pessoal do terapeuta em levar o cliente a refletir sobre o que ele traz para a psicoterapia, ajudando-o a pensar em como buscar o que é melhor para si. Então, a terapia recria o contexto que o filósofo Martin Buber se referia que o ser humano é um ser de relação e é nestas condições que ele se desenvolve (Amatuzzi, 2012).

\section{A relação não-hierárquica entre cliente e psicólogo na Abordagem Centrada na Pessoa}

O psicoterapeuta, na Abordagem Centrada na Pessoa, usa seus sentimentos como movimentações direcionadas ao cliente, intercorrendo uma forma íntima de entendimento entre ambos (Moreira, 2010a). Para Rogers (2001), a mudança e o progresso pessoal do cliente são favorecidos quando o psicoterapeuta demonstra o que é de fato, ou seja, quando na sua relação ele é autêntico e não há disfarces, podendo manifestar de forma direta suas ações e sentimentos na hora em que acontecem. A palavra congruência é utilizada para nomear esta atitude.

A congruência, neste caso, significaria que os sentimentos vivenciados pelo psicoterapeuta estão acessíveis à sua consciência e ele possui a capacidade de expressá-los quando for oportuno. Nesta fase, a prática clínica da ACP se aproxima da fenomenologia, pois muda o foco do cliente para a sua experiência interpessoal (Rogers, 2001).

Rogers (1983) levanta a hipótese de que há no universo uma força que o direciona de maneira formativa e ela está presente e pode ser visualizada nos microrganismos, no espaço estelar, nas vidas orgânicas mais complexas e também no ser 
humano. No ser humano, esta tendência se manifesta no momento em que o sujeito, partindo de sua origem unicelular, avança para um desempenho orgânico complexo, para uma forma de sentir e conhecer descensional ao grau da consciência, para um saber lúcido do mundo exterior e do seu organismo, para um estado consciente que transcende, e é de unicidade com o sistema cósmico e também de equilíbrio, no qual se encontra o ser humano.

Desta forma, o papel do psicólogo na ACP não precisaria ser de um condutor para aquele indivíduo, alguém que detém o saber sobre ele e sobre verdades universais e, por isso, está em uma posição hierarquicamente mais alta. Sua tarefa é ser um facilitador que caminharia lado a lado com o cliente que possui uma autonomia para entender quais as melhores escolhas para seu contexto e possui em si um potencial autorealizador.

A tendência atualizante é definida como uma probabilidade intrínseca e particular de um indivíduo, sendo uma tendência orgânica ao seu desenvolvimento. De acordo com Rogers (1977), este tipo de sujeito consegue exercer plenamente suas potencialidades e não para nunca de progredir. A ideia de organismo é o núcleo de sua teoria; a maneira como funciona o organismo ocorre por meio da tendência atualizante que faz surgir uma grande habilidade de aprender e criar no sujeito, quando ele está em um contexto facilitador. A tendência atualizante, destaca Moreira (2010b), pode ser uma das bases da relação não-hierárquica entre cliente e psicólogo.

Rogers, ao longo de sua obra, coloca que o objetivo do terapeuta é participar da experiência imediata do seu cliente. Para isto é necessário que o terapeuta saiba escutar e observar, estar atento aos movimentos da relação e à sua interação com seu cliente. Na ótica rogeriana, o principal conceito - que rege a quase totalidade dos processos envolvidos tanto na clínica, quanto no cotidiano de seus clientes - é o de "tendência atualizante". (Holanda, 2009, p. 42)

O psicoterapeuta ao proporcionar um ambiente facilitador dentro do contexto terapêutico pode proporcionar com que a tendência atualizante do cliente seja utilizada. Desta forma, não necessitará conduzir as sessões de modo rígido, nem realizar interpretações pelo cliente e muito menos estar em uma posição de detentor de todo o saber.

Com relação ao desenvolvimento psicoterapêutico:

A importância das condições facilitadoras, propostas por Rogers, e, entre elas, a compreensão empática, se mantém na prática clínica de psicoterapeutas humanista-fenomenológicos iniciantes. Mas aqui elas estão acrescidas, ou densificadas, pelas técnicas fenomenológicas de intervenção (a intuição eidética, a redução fenomenológica, a descrição, a fala autêntica e ver e ouvir fenomenologicamente), tais como propostas por Moreira (2009; 2012), inspiradas na fenomenologia filosófica de Merleau-Ponty, propiciando a tentativa de acesso ao mundo vivido do outro (Fontgalland et al., 2018, p. 17).

Para Vieira e Freire (2006), é importante visar ainda que a tendência atualizante não apresenta uma forma de equilíbrio constante, pois ela movimenta uma série de adaptações de padrões no sujeito. A tendência atualizante será, então, direcionada a ampliar a geração de padrões e conhecimentos novos.

Umas das inferências práticas do conceito de tendência atualizante se encontra na questão de que o cliente se torna sujeito da sua vida, sendo assim, ele é consciente e também ativo. Frente a isto, ao analisar estas inferências na clínica, precisamos visualizar a concepção de homem, e, consequentemente, a possível alteração na posição inicial do psicoterapeuta neste relacionamento. Se o indivíduo que procura a psicoterapia possui autonomia, consciência e as potencialidades necessárias para se desenvolver, a função atribuída ao psicólogo não é mais de detentor do saber ou ainda de um guia (Holanda, 2009).

Holanda (2009) esclarece que ocorre um nivelamento de posição entre cliente e psicoterapeuta, pois ambos são humanos, e nisto se firma a simplicidade da clínica psicológica de Rogers. Neste ambiente, é retirado do psicoterapeuta o entendimento de sua figura como algo mágico e ainda responsável pelo processo terapêutico que passa a ser do cliente, o que 
fundamenta o uso da palavra 'facilitador' em vez de 'terapeuta', por Rogers. Isto também espelha a atitude do que Rogers chamou de facilitador, ou seja, a sua atitude durante a entrevista demonstra sua convicção na tendência atualizante.

Portanto, Rogers (2001) destaca a necessidade que psicólogo seja de fato o que se é. Sendo assim, por acreditar que o mais fundamental do processo psicoterapêutico é aumentar as potencialidades do cliente, o facilitador evita se utilizar de atitudes 'diretivas' e de ser o gestor principal do processo terapêutico de seu cliente, estando em um lugar de facilitar a urgência do fenômeno dele (Holanda, 2009).

\section{Considerações Finais}

O objetivo deste estudo foi de compreender a posição horizontal e não-hierárquica entre psicoterapeuta e cliente na Abordagem Centrada na Pessoa na clínica psicológica. Foi possível elucidar o funcionamento da clínica psicológica na ACP e como ela lida com a relação não-hierárquica entre cliente e psicoterapeuta, também chamado de facilitador. Foi também possível conhecer sobre o contexto histórico da palavra clínica e a clínica psicológica.

O processo terapêutico na ACP é rico e apresenta uma proposta para o cliente, ou seja, que ele possa assumir o papel de ser o dono e condutor deste processo, enquanto o psicoterapeuta atua como um facilitador durante a jornada. Sugere-se, que mais estudos sejam elaborados sobre a ACP e suas relações com o cliente na clínica, sendo que, no momento contemporâneo, é uma possibilidade investigar como a clínica psicológica tem se adaptado ao atendimento online e o distanciamento social devido a pandemia de Covid-19.

\section{Referências}

Aguirre, A. B., Herzberg, E. Pinto, E. B., Becker E., Carmo, H. M. S., \& Santiago, M. D. E. (2000). A formação da atitude clínica no estagiário de psicologia. Psicologia USP, 11(1), 49-62. https://doi.org/10.1590/S0103-65642000000100004

Amatuzzi, M. M. (2012). Rogers: Ética Humanista e Psicoterapia. Alínea.

Araujo, I. C., \& Freire, J. C. (2014). Os valores e a sua importância para a teoria da clínica da abordagem centrada na pessoa. Revista da Abordagem Gestáltica, 20(1), 86-93. http://pepsic.bvsalud.org/scielo.php?script=sci_arttext\&pid=S1809-68672014000100012

Calabria, A. C. \& Kempfer, A. V. (2020). Comunicação não-violenta: desenvolvimento na prática e artigo de atualização. Research, Society and Development, 9(12), e40191211142. http://dx.doi.org/10.33448/rsd-v9i12.11142

Compas, B., \& Gotlib, E. (2003). Introducción a la Psicología Clínica: Ciencia y práctica. Mc.Graw Hill.

Fontgalland, R. C., Moreira, V., \& Melo, C. F. (2018). A experiência de ser empático para o psicoterapeuta humanista-fenomenológico iniciante. Arquivos Brasileiros de Psicologia, 70(1), 5-20. http://pepsic.bvsalud.org/scielo.php?script=sci_arttext\&pid=S1809-52672018000100002

Foucault, M. (1977). O nascimento da clínica. Forense Universitária.

Gil, A. C. (2002). Como elaborar projetos de pesquisa. Atlas.

González, H. (2008). Um paseo por la historia de la psicologia clínica y de la salud: entrevista a Helio Carpintero. Clínica y Salud,18(1), 121-129.

Holanda, A. F. (2009). A perspectiva de Carl Rogers acerca da resposta reflexa. Revista do NUFEN, 1(1), 40-59. http://pepsic.bvsalud.org/scielo.php?script=sci_arttext\&pid=S2175-25912009000100004

Magalhães, M., Straliotto, M., Keller, M., \& Gomes, W. B. (2001). Eu quero ajudar as pessoas: a escolha vocacional da psicologia. Psicologia: ciência e profissão, 21(2), 10-27. http://pepsic.bvsalud.org/scielo.php?script=sci_arttext\&pid=S1414-98932001000200003\&lng=pt\&nrm=iso\&tlng=pt

Moreira, J. O., Romagnoli, R. C., \& Neves, E. O. (2007). O surgimento da clínica psicológica: da prática curativa aos dispositivos de promoção da saúde. Psicologia: ciência e profissão, 27(4), 608-621. https://doi.org/10.1590/S1414-98932007000400004

Moreira, V. (2010a). Convergências e divergências entre as psicoterapias de Carl Rogers e Frederick Perls. Revista do NUFEN, 2(1), 20-50. http://pepsic.bvsalud.org/scielo.php?script=sci_arttext\&pid=S2175-25912010000100003

Moreira, V. (2010b). Revisitando as fases da abordagem centrada na pessoa. Estudos de Psicologia (Campinas), 27(4), 537-544. https://doi.org/10.1590/S0103-166X2010000400011

Moreira, V., \& Torres, R. B. (2013). Empatia e redução fenomenológica: possível contribuição ao pensamento de Rogers. Arquivos Brasileiros de Psicologia, 65(2), 181-197. http://pepsic.bvsalud.org/scielo.php?script=sci_arttext\&pid=S1809-52672013000200003 
Research, Society and Development, v. 11, n. 2, e2411225307, 2022

(CC BY 4.0) | ISSN 2525-3409 | DOI: http://dx.doi.org/10.33448/rsd-v11i2.25307

Moreno, J. E. C. (2014). Psicología clínica: revisión contextual y conceptual. Revista Electrónica Psyconex. 6(9), 1-20. https://revistas.udea.edu.co/index.php/Psyconex/article/view/22435/18573

Rogers, C. (1977). Definições das noções teóricas. In C. Rogers \& M. Kinget (Eds), Psicoterapia e relações humanas, 1, 157-179. Interlivros.

Rogers, C. (1983). Um jeito de ser. E.P.U.

Rogers, C. (1992). Terapia Centrada no Cliente. Martins Fontes.

Rogers, C. (2001). Tornar-se pessoa. Martins Fontes.

Scorsolini-Comin, F. (2014). Aconselhamento psicológico e psicoterapia: aproximações e distanciamentos. Contextos Clínicos, 7(1), 02-14. http://pepsic.bvsalud.org/scielo.php?script=sci_arttext\&pid=S1983-34822014000100002

Trull, T. \& Phares, E. (2003). Psicología clínica. Conceptos, métodos y aspectos prácticos de la profesión. Cengage Learning.

Vieira, E. M., \& Freire, J. C. (2006). Alteridade e Psicologia Humanista: uma leitura ética da abordagem centrada na pessoa. Estudos de Psicologia (Campinas), 23(4), 425-432. https://doi.org/10.1590/S0103-166X2006000400010 